International Mathematical Forum, 2, 2007, no. 34, 1657 - 1701

\title{
Survey of Power, QR, and Iterative Methods for Solution of Largest Eigenvalue of Essentially Positive Matrices
}

\section{Tedja Santanoe Oepomo}

Science, Engineering, and Mathematics Division, West LA College, Riverside

Community College, Los Angeles Harbor College, and ITT-Technical Institute-

Sylmar, CA, 945 South Idaho Units: 134, Las Habra, Ca 90631, USA

oepomots@elac.edu, oepomot@wlac.edu

\begin{abstract}
Many of the popular methods for the solution of largest eigenvalue of essentially positive irreducible matrices are surveyed with the hope of finding an efficient method suitable for electromagnetic engineering, radiation problems, system identification problems, and solid mechanics. Eigenvalue computations are both fundamental and ubiquitous in computational science and its fast application areas. Some comparisons between several known algorithms, i.e. Power and QR methods, and earlier theory of Oepomo iterative techniques for solving largest eigenvalue of nonnegative irreducible matrices are presented since there is a continuing demand for new algorithm and library software that efficiently utilize and adapt to new applications.
\end{abstract}

Keywords, Collatz's theorem, Perron-Frobernius' theorem, and eigenvalue 
Mathematics Subject Classification: 15A48, 15A18, 15A99

Introduction. Without doubt the power and QR iterative methods have proved most popular among applied mathematician-that is if popularity is measured by frequency of use. In our discussions of the various methods and the results of comparisons with Oepomo methods, only references that are directly relevant are noted. No attempt has been made to cite the earliest sources.

Eigenvalue Computation. The second fundamental problem in linear algebra is to compute the eigenvalues and eigenvectors of a square matrix. In other words, to diagonalize a square matrix. In theory we know what to do. We have to compute the characteristic polynomial $P(\lambda)$, find the roots $\lambda_{i}$, then for each $i$ we have the equation $\left(A-\lambda_{i}\right) x=0$ for the eigenvectors. However, this procedure is not satisfactory.

Newtown's method give a very fast way to compute roots of polynomial $P(\lambda)$, it can also easily be extended to complex roots. But remember, that Newton's method usually works very well once you are reasonably "close" to the root, but it may diverge if you start far away. Still you need ad hoc method to get "close" to the root. To reduce the chance element in this "hit or miss" strategy, another numerical computations were invented, i.e. power, QR iterative, and Oepomo’s methods. 
Moreover, we also have to care about the stability of the method, as the eigenvalue problem can be quite ill conditioned, so we do not want to make it much worse by an unstable algorithm.

Example. Consider the nxn matrix

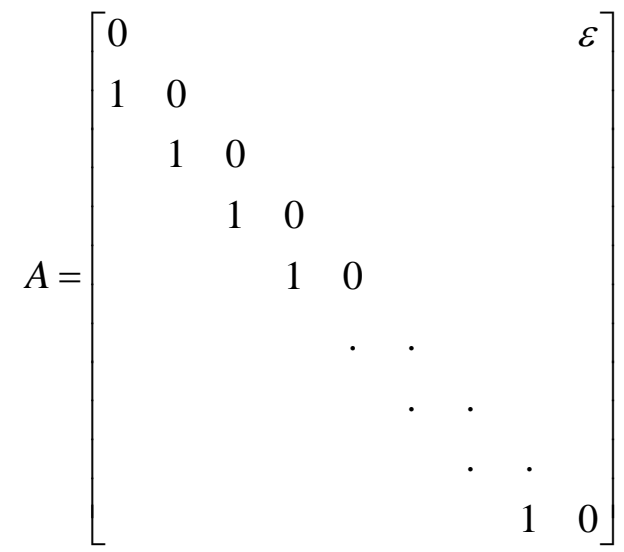

where $\varepsilon$ is a tiny number with all entries are zero. The characteristic polynomial of $A$ is $P(\lambda)=\lambda^{n}-\varepsilon$. Clearly all eigenvalues are 0 if $\varepsilon=0$. However for $\varepsilon \neq 0$ there are $n$ complex eigenvalues, the $n$-th roots of unity. Even if we focus on the real eigenvalue $\varepsilon^{1 / n}$, it is clear that the problem is very ill conditioned. Let $\mathrm{n}=40$ and choose $\varepsilon=10^{-40}$ which is an extremely tiny relative error of order $\frac{10^{-40}}{1}=10^{-40}$. However, one of the eigenvalue is $\lambda=10^{-1}=0.1$, which is at a distance of $10^{-1}$ from the "unperturbed" eigenvalue of 0 . Hence the change in the eigenvalues is equal to 
the change in the perturbation parameter $\varepsilon$ multiplied by $10^{+39}$. There is another disquieting aspect of this phenomenon. The number $\varepsilon=10^{-40}$ is automatically replaced by 0 in the computer, and this rounding introduces an error of order $10^{-1}$ in the result.

Fortunately the eigenvalue problem is not so ill conditioned for "most" matrices, this example were particularly nasty one. However, it shows that we should aim at stable algorithms, which at least do not make "bad things much worse", since the problem itself can be quite "bad".

Power Method. This method is extremely simple. Let $A$ be a square matrix. Pick any vector $x_{0}$ and start successively multiplying it with $A$. We can claim, that unless you are extremely unlucky with the choice of $x_{0}$, we can easily find the eigenvalue with the largest modulus.

Theorem 1: Suppose that the square matrix $A$ has one eigenvalue $\lambda_{1}$ that is greater in absolute value than all other eigenvalues, and let $x_{n+1}=A x_{n}$. Then from randomly chosen nonzero vectors $x_{0}$ and $w$ we have $\quad \lim _{n \rightarrow \infty} \frac{w^{t} \bullet x_{n+1}}{w^{t} \bullet x_{n}}=\lambda_{1}$ 
"almost surely". Moreover, the sequence of normalized vectors $\tilde{X}_{n}:=\frac{x_{n}}{\left\|x_{n}\right\|}$ converges to the eigenvector of $\lambda_{1}$.

Remark. The probabilistic expression "almost surely" in the theorem can be made mathematically precise, i.e. it means "for almost all" vector, with the exception of vectors lying in a smaller subspace, but we do not want to go into this detail. For the present purpose of the manuscript you should take it literally.

Proof: We give a proof only for a case where $A$ is symmetric $k \times k$ matrix i.e. $A=V D V^{t}$, and $\lambda_{1}$ is a simple eigenvalue. The proof for non-symmetric diagonalizable matrices is slightly harder, and the case of a general matrix $A$ is moderately harder. We can use the spectral theorem, i.e.

$$
A=\sum_{i=1}^{k} \lambda_{i} v_{i} v_{i}^{t}
$$

clearly $\quad A^{n}=\sum_{i=1}^{k} \lambda_{i}^{n} v_{i} v_{i}^{t}$ and $\quad w^{t} \bullet x_{n}=w^{t} \bullet A^{n} x_{0}=\sum_{i=1}^{k} \lambda_{i}^{n}\left(w^{t} \bullet v_{i}\right)\left(v_{i}^{t} \bullet x_{0}\right)$

We compute $\lim _{n \rightarrow \infty} \frac{w^{t} \bullet x_{n}}{\lambda_{1}^{n}}=\lim _{n \rightarrow \infty} \sum_{i=1}^{k}\left(\frac{\lambda_{i}}{\lambda_{1}}\right)^{n}\left(w^{t} \bullet v_{i}\right)\left(v_{i}^{t} \bullet x_{0}\right)=\left(w^{t} \bullet v_{1}\right)\left(v_{1}^{t} \bullet x_{0}\right)$

since $\left|\lambda_{1}\right|>\left|\lambda_{i}\right|$ for $\mathrm{i}>1$. Assume that $x_{0}$ and $w$ are not orthogonal to $v_{1}$ (this is the case that one has to exclude by choosing the vectors "randomly”). Then 


$$
\lim _{n \rightarrow \infty} \frac{w^{t} \bullet x_{n+1}}{w^{t} \bullet x_{n}}=\lambda_{1} \frac{\lim \frac{w^{t} \bullet x_{n+1}}{\lambda_{1}^{n+1}}}{\lim \frac{w^{t} \bullet x_{n}}{\lambda_{1}^{n}}}=\lambda_{1} \frac{\left(w^{t} \bullet v_{1}\right)\left(v_{1}^{t} \bullet x_{0}\right)}{\left(w^{t} \bullet v_{1}\right)\left(v_{1}^{t} \bullet x_{0}\right)}=\lambda_{1}
$$

completes the proof of the eigenvalue convergence. To see the convergence of the normalized eigenvectors, notice that

$$
\begin{aligned}
& x_{n}=A^{n} x_{0}=\sum_{i=1}^{k} \lambda_{i} v_{i}\left(v_{i}^{t} \bullet x_{0}\right) \text { hence }\left\|x_{n}\right\|=\sqrt{\sum_{i=1}^{k}\left|\lambda_{i}\right|^{2 n}\left(v_{i}^{t} \bullet x_{0}\right)^{2}} \text {, so } \\
& \tilde{X}_{n}=\frac{x_{n}}{\left\|x_{n}\right\|}=\frac{\sum_{i=1}^{k} \lambda_{i}^{n}\left(v_{i}^{t} \bullet x_{0}\right)^{2}}{\sqrt{\sum_{i=1}^{k}\left|\lambda_{i}\right|^{2 n}\left(v_{i}^{t} \bullet x_{0}\right)^{2}}}=\left(\frac{\lambda_{1}\left(v_{1}^{t} \bullet x_{0}\right)}{\mid \lambda_{1}\left(v_{1}^{t} \bullet x_{0}\right)}\right)^{n} \frac{v_{1}+\sum_{i=2}^{k}\left(\frac{\lambda_{i}}{\lambda_{1}}\right)^{n} \frac{v_{i}^{t} \bullet x_{0}}{v_{1}^{t} \bullet x_{0}} \bullet v_{i}}{\sqrt{1+\sum_{i=2}^{k}}\left|\frac{\lambda_{i}}{\lambda_{1}}\right|^{2 n} \frac{\left(v_{i}^{t} \bullet x_{0}\right)^{2}}{\left(v_{1}^{t} \bullet x_{0}\right)^{2}}}
\end{aligned}
$$

and if $\left|\lambda_{1}\right|>\left|\lambda_{i}\right|$ for all other $i$, then this clearly converges $v_{1}$.

This method gave only the eigenvalue of largest modulus. With a little trick, one can get all other eigenvalues,

Theorem 2 Suppose that the square matrix $A$ has an eigenvalue $\lambda_{p}$, which is closer to $p$ than all other eigenvalues. Run the

$$
x_{n+1}=(A-p I)^{-1} x_{x}
$$


iteration with some initial vector $x_{0}$. If the vectors $x_{0}$ and $w$ are chosen randomly, then

$$
\begin{aligned}
& \lim _{n \rightarrow \infty} \frac{w^{t} \bullet x_{n+1}}{w^{t} \bullet x_{n}}=\frac{1}{\lambda_{p}-p} \text { “almost surely”. Hence } \lambda_{p} \text { is obtained as } \\
& \lambda_{p}=\lim _{n \rightarrow \infty} \frac{w^{t} \bullet x_{n}}{w^{t} \bullet x_{n+1}}+p \quad\left(\text { if } \quad \lambda_{p} \neq p\right) . \quad \text { Moreover, again, the }
\end{aligned}
$$

normalized vectors $\tilde{X}_{n}:=\frac{x_{n}}{\left\|x_{n}\right\|}$ converge to the eigenvector belonging to $\lambda_{p}$.

Proof: Simply that the eigenvalues of $B:=(A-p I)^{-1}$ are $\left(\lambda_{1}-p\right)^{-1},\left(\lambda_{2}-p\right)^{-1}, \ldots \ldots$, where $\lambda_{i}$ are the eigenvalues of $A$. Hint: this statement does not require $A$ being symmetric, so spectral theorem cannot be used. From the condition it follows that $\left(\lambda_{1}-p\right)^{-1}$ is the largest in modulus among them, so we can apply Theorem 1 . The statement on the eigenvector is straightforward from Theorem 1.

Remark: The power method looks very simple and elegant. However, notice it is really powerful only for the eigenvalue largest in modulus. Applying Theorem 2 already requires inverting a matrix, and more importantly it requires knowing a point $p$ “near" the eigenvalue. Hence we run into similar difficulties when we applied 
Newtown's iteration for finding the roots of the characteristics polynomial. In fact there is essentially a Newtown's iteration behind Theorem 2 .

For iterative Power algorithm let $A$ be an (n, n) matrix. Let $\lambda_{1}, \lambda_{2}, \ldots \ldots \ldots, \lambda_{n}$ be the eigenvalues of $A$ and let

$$
\left|\lambda_{1}\right|=\left|\lambda_{2}\right|=\ldots \ldots \ldots . . .\left|\lambda_{k}\right|>\left|\lambda_{k+1}\right| \geq \ldots \ldots . . \geq\left|\lambda_{n}\right|
$$

and $\lambda_{1}=\lambda_{2}=\ldots \ldots . .=\lambda_{k}$ for $1 \leq k \leq n$.

We shall refer to the eigenvalues $\lambda_{1}, \lambda_{2}, \ldots \ldots . . ., \lambda_{k}$ as dominant eigenvalues and to the corresponding eigenvectors as the dominant eigenvectors.

Let $v_{0}$ be a linear combination of the eigenvectors. In the power method we let $A$ operate repeatedly on the vector $v_{0}$. In essence, the $\mathrm{m}^{\text {th }}$ iterated vector is given by

$$
v_{m}=A^{m} v_{0}
$$

Apart from exceptional cases,

$$
\lim _{m \rightarrow \infty}\left(\frac{1}{\lambda^{m}}\right) A^{m} v_{0}=x_{1}
$$

is the dominant eigenvector.

The convergence of the power method can be proven when the elementary divisor of $A$ is non-linear. Detail of this can be found in [12, p. 582]. Here we will prove the 
convergence of the power method with the assumption that the elementary divisors of $A$ are linear [2, p. 226]. Let $x_{1}, x_{2}, \ldots \ldots \ldots, x_{n}$ be $\mathrm{n}$ linearly independent eigenvectors of $A$ corresponding to $\lambda_{1}, \lambda_{2}, \ldots \ldots . ., \lambda_{k}$ respectively. Let the dominant eigenvalue be of multiplicity $\mathrm{k}$. We assume that the vector $v_{0}$ is "sufficiently rich" in $x_{1}$ i.e. $v_{0}$ has not too small component in the $x_{1}$ direction. This is a rather mild condition, as during the iteration round off error may anyway introduce a spurious component in the $x_{1}$ direction. We will express $v_{0}$ as a linear combination of the eigenvectors

$$
v_{0}=\sum_{i=1}^{n} \alpha_{i} x_{i}
$$

Equation (5) is multiplied by $A$ repeatedly and in general the $\mathrm{m}^{\text {th }}$ iterated vector is given by

$$
\begin{aligned}
v_{m}=A^{m} v_{0}=\sum_{i=1}^{n} A^{m} \alpha_{i} x_{i} & =\sum_{i=1}^{n} \alpha_{i} \lambda_{i}^{m} x_{i}=\lambda_{1}^{m} \sum_{i=1}^{k} \alpha_{i} x_{i}+\sum_{i=k+1}^{n} \lambda_{i}^{m} \alpha_{i} x_{i} \\
& =\lambda_{1}^{m}\left[\sum_{i=1}^{k} \alpha_{i} x_{i}+\sum_{i=k+1}^{n}\left(\frac{\lambda_{i}}{\lambda_{1}}\right)^{m} \alpha_{i} x_{i}\right]
\end{aligned}
$$


remembering that the dominant eigenvalue is of multiplicity k. For $m \rightarrow \infty$ the term in the bracket converges to $\sum_{i=1}^{k} \alpha_{i} x_{i}$. The sequence converges to an eigenvector corresponding to $\lambda_{1}$.

$$
\lim _{m \rightarrow \infty}\left(\frac{1}{\lambda_{1}^{m}}\right) A^{m} v_{0}=\sum_{i=1}^{k} \alpha_{i} x_{i} \text {. It follows from (6) } \lambda_{1}=\lim _{m \rightarrow \infty} \frac{\left(A^{m+1} v_{0}\right)_{r}}{\left(A^{m} v_{0}\right)_{r}}
$$

Power algorithm:

1. Choose an initial vector $y_{0}$.

2. Given a vector $y_{k}$ the vectors $y_{k+1}$ and $z_{k+1}$ are formed as follows

$$
z_{k+1}=A y_{k} \text { and } y_{k+1}=\frac{z_{k+1}}{C_{k+1}}
$$

where $c_{k+1}=\max _{i}\left|\left(z_{k+1}\right)_{i}\right|$.

The introduction of $c_{k+1}$ in (7) will prevent $z_{k}$ from being too large. Successive approximations to $\lambda_{1}$ are computed as the ratio of $\frac{\left(z_{k+1}\right)_{r}}{\left(y_{k}\right)_{r}}$. 
Rate of convergence: Let the eigenvalues of the given matrix be defined in (2). The rate of convergence of the power method depends on how fast the ratio of $\left(\frac{\left|\lambda_{k+1}\right|}{\left|\lambda_{k}\right|}\right)^{m}$ goes to zero.

Discussions: The power method converges very slowly if the separation between $\lambda_{k+1}$ and $\lambda_{1}$ is poor; in such cases it may be feasible to speed up the convergence as follows. Consider the matrix $A-q I$ where $q$ is a number and $\mathrm{I}$ is the identity matrix. The matrix $A-q I$ has eigenvalues $\lambda_{i}-q$, and if $q$ is chosen properly, convergence to an eigenvector may be sped up. If, for example, $\lambda_{1}>\lambda_{2} \geq \ldots \ldots \ldots \geq \lambda_{n}>0$, then the optimum value of $q$ for the maximum rate of convergence is $\left(\frac{\lambda_{2}+\lambda_{n}}{2}\right)$. Of course we need some knowledge of the eigenvalues to apply this method, but if the eigenvalues are known to be positive, then trial values of $q$ can be easily chosen to find the value yielding the best rate of convergence. This is the shift of origin technique and has proven to be effective for some eigenvalue distribution [12, p. 572]. 
If $\lambda$ is an eigenvalue of $A$ then $\lambda^{-1}$ is an eigenvalue of $A^{-1}$. According to a variant of the power method that may be called the inverse power algorithm, sequences $\left\{x^{m}\right\}\left\{y^{m}\right\}$ are formed by the following recursion
(i) $x^{m+1}=A^{-1} y^{m}$
(ii) $y^{m+1}=\frac{x^{m+1}}{\max \left|x_{i}^{m+1}\right|}$

Each step here requires the solution of the linear system $A x^{m+1}=y^{m}$.

Using the inverse power method we can find the absolutely smallest eigenvalue and the corresponding eigenvector of the matrix.

The inverse power method can be considerably accelerated by the shift of origin technique, if $A$ is a Stieltjes matrix. $A$ real $(n, n)$ matrix $A=\left(a_{i j}\right)$ with $a_{i j} \leq 0$ for all $\mathrm{i} \neq \mathrm{j}$ is a Stieltjes matrix if $A$ is symmetric and positive definite. Let the eigenvalue of $A$ be given by $0<\lambda_{1} \leq \lambda_{2} \leq \ldots \ldots . . . \lambda_{n}$. If $A$ is irreducible Stieltjes matrix it can be proven [11, p. 288] that for all $0<\sigma_{m}<\lambda_{1}$ (for $\mathrm{m}=0,1, \ldots$ ).

$$
\left(A-\sigma_{m} I\right)^{-1}>0
$$

$A^{-1}$ has the same eigenvectors as $\left(A-\sigma_{m} I\right)^{-1}$, therefore we replace in (i) $A^{-1}$ by $\left(A-\sigma_{m} I\right)^{-1}$ and we get the recursive relation 


$$
\text { (i) } x^{m+1}=\left(A-\sigma_{m I}\right)^{-1} y^{m} ; \quad \text { (ii) } y^{m+1}=\frac{x^{m+1}}{\max \left|x_{i}^{m+1}\right|}
$$

where $\sigma_{0}=0$ and $y^{0}>0$ is the initial value of starting vector. Using Collatz's theorem to the last relation it can be shown [1, p. 288] that

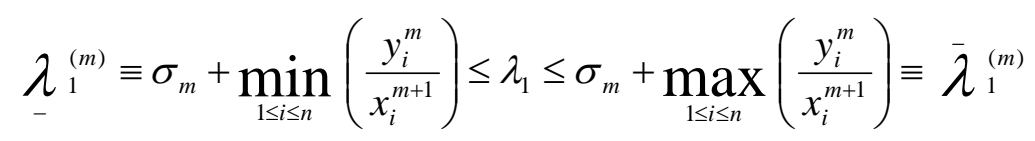

where the first and the last terms are lower and upper bounds for $\lambda_{1}$. If equality holds in the above equation than $\lambda_{1}^{(m)}=\bar{\lambda}_{1}^{(m)}=\lambda_{1}$, and the procedure terminates. On the other hand if these bounds are unequal we define the following relationship $\sigma_{m+1}=\lambda_{1}^{(m)}$ and continue the iterative process.

QR Iteration for Eigenvalues: We will discuss the QR algorithm, which is the most frequently used for calculation of the set of eigenvalues of a general matrix. However, it does not compute eigenvectors unless the matrix is symmetric. The QR method seeks the reduction of e general matrix to a triangular from with the aid of unitary transformation. Instead of the triangular decomposition, as in LR method, the QR method uses the factors of the type

$$
A=Q R
$$


where $Q$ is a unitary matrix and $R$ an upper triangular matrix.

Theorem 3 Schur's theorem, every non-singular matrix can be factored in the form (8).

Proof: [6, pp. 142]

If $A$ is non-singular, it will be shown that the $Q R$ decomposition is unique, apart from a diagonal multiplier having elements of modulus unity.

Let $Q_{1} R_{1}$ and $Q_{2} R_{2}$ be the $Q R$ decomposition of a non-singular matrix $A$.

$$
A=Q_{1} R_{1}=Q_{2} R_{2} \text {. Then, } \operatorname{Det}(A)=\operatorname{det}\left(Q_{1}\right) \operatorname{det}\left(R_{1}\right)=\operatorname{det}\left(Q_{2}\right) \operatorname{det}\left(R_{2}\right) \text {. }
$$

The non-singularity of $R_{1}$ and $R_{2}$ follows from that of $A$. Then, $Q_{2}^{H} Q_{1}=R_{2} R_{1}^{-1}=R^{1}$

where $\mathrm{H}$ denotes the conjugate transpose, and $R^{1}$ is upper triangular. Taking the conjugate transpose of the above equation, $Q_{1}^{H} Q_{2}=\left(R^{1}\right)^{H}$. Therefore, $\left(R^{1}\right)^{H} R^{1}=I$ From the above equation, it follows that $R^{1}$ is also unitary. Equating elements on both sides, it can be easily seen that $R^{1}$ is a diagonal matrix, such that the element of $R^{1}$ are given by

$$
\left|r_{i j}^{1}\right|=1 \text {. Therefore, }
$$




$$
\begin{aligned}
& Q_{2}^{H} Q_{1}=R_{2} R_{1}^{-1}=R^{1} D, \\
& Q_{2}=Q_{1} D^{H} ; \quad \text { where } \mathrm{D} \text { is a diagonal matrix with modulus unity's } \\
& R_{2}=D R_{1}
\end{aligned}
$$

elements. Thus $Q R$ decomposition given in (8), is unique apart from a diagonal multiplier having elements of modulus unity. We can always chose D such that $\left(R_{2}\right)_{i i}>0$. Then the $Q_{2} R_{2}$ decomposition will be considered normalized. On the other hand, this normalized decomposition is clearly unique. Hence forth, whenever a $Q R$ 's decomposition is formed, we will assume that the diagonal elements of $R$ are real positive.

In the $Q R$ algorithm, we start from a given matrix $A=A_{1}$ and from sequences of matrices $A_{K}, Q_{K}$, and $R_{K}$ by the use of

$$
\begin{aligned}
& A_{K}=Q_{K} R_{K} \text { for } \mathrm{k}=1,2,3, \ldots . \\
& A_{k+1}=R_{k} Q_{k}=Q_{k}^{H} A_{k} Q_{k}=Q_{k+1} R_{k+1}
\end{aligned}
$$

It can be proved under certain condition that $\lim _{k \rightarrow \infty} A_{k}$ is an upper triangular matrix.

The proof of the convergence of a generalized form of the $Q R$ method, when some eigenvalues are complex conjugate and when the elementary divisors are nonlinear, can be found in $[13,10]$. If pairs of eigenvalues of equal modulus are allowed the 
sequence of $A_{k}$ is not convergent. For sufficiently large $k, A_{k}$ is close to a matrix $\bar{A}_{k}$ of block upper triangular structure. Each diagonal block $(1,1)$ or $(2,2)$. The eigenvalues of $\bar{A}_{k}$ are those of the diagonal blocks. For sufficiently large $k$ the eigenvalues and eigenvectors of $\bar{A}_{k}$ are good approximation of those of $A_{k}$. The proof of the convergence of the $Q R$ method can be found in $[13,5]$. The following assumption is made:

The eigenvalues of $A$ are of distinct modulus, say, $\left|\lambda_{1}\right|>. \lambda_{2} .>\ldots \ldots>\left|\lambda_{n}\right|$.

The proof of the convergence of the QR algorithm follows very similar line to those of LR algorithm. Using (9a) and (9b), it can be shown by induction that

$$
A_{k+1}=\xi_{k}^{H} A_{1} \zeta_{k}
$$

where

$$
\zeta_{k}=Q_{1} Q_{2} \ldots \ldots . . Q_{k} .
$$

Thus $A_{k+1}$ is unitarily similar to $A_{1}$. Then, as in the derivation of LR algorithm,

$$
\xi_{k} \mathcal{R}_{k}=\left(Q_{1} R_{1}\right)^{k}=A_{1}^{k}
$$

where

$$
R_{k}=R_{k} R_{k-1} \ldots \ldots R_{1}
$$

Using (11), and as the elementary divisors of $A$ are linear, we can write 


$$
\xi_{k} \mathcal{R}_{k}=A_{1}^{k}=X D^{k} X^{-1}=X D^{k} Y^{T}
$$

where $X$ and $Y^{T}$ are matrices formed by the right hand and left hand eigenvectors of $A$, and $D$ is the diagonal matrix. WE will now consider the triangular decomposition of matrix $P Y$, defined in connection with (12), and remembering that $P$ is a permutation matrix from (12),

$$
\xi_{k} \mathcal{R}_{\mathrm{k}}=X D^{k} P^{T} P Y=X D^{k} P^{T} L_{y} R_{y}=X P^{T} \tilde{D^{k}} L_{y} R_{y}
$$

where $\tilde{D^{k}}=P D^{k} P^{T}$ is a diagonal matrix, the element of which are in the order $\lambda_{i_{1}}, \lambda_{i_{2}}, \lambda_{i_{3}}, \ldots \ldots, \lambda_{i_{n}}$. Let

$$
X P^{T}=Q_{X} R_{x}
$$

where $Q_{x}$ is a unitary matrix and $R_{x}$ is an upper triangular matrix. From (13) and (14),

$$
\begin{aligned}
\xi_{k} R_{k}= & Q_{x} R_{x} \tilde{D}^{k} L_{y} R_{y}=Q_{x} R_{x} \tilde{D}^{k} L_{y}\left\{\tilde{D}^{-k} \tilde{D}^{k}\right\} R_{y} \\
= & Q_{x} R_{x}\left\{\tilde{D^{k}} L_{y} D^{-k}\right\} \tilde{D}^{k} R_{y} .
\end{aligned}
$$

Let $\quad D^{k} L_{y} D^{-k}=\mathrm{I}+F_{k}$. 
$F_{k}$ is a lower triangular matrix, the element $(r, s)$ of which is given by [13] in the LR method. Just as in LR method,

$$
F_{k} \rightarrow 0 \text { as } \mathrm{k} \rightarrow \infty \text {. Hence, we obtain from (15), } \xi_{k} \mathbb{R}_{k}
$$

$=Q_{x} R_{x}\left(I+F_{k}\right) \tilde{D^{k}} R_{y}$.

Following derivation of LR method in [13], we get

$$
\xi_{k} \mathcal{R}_{k}=Q_{x}\left(I+E_{k}\right) R_{x} \tilde{D}^{k} R_{y}
$$

where $E_{k}=R_{x} F_{k} R_{x}^{-1}$, and $E_{k} \rightarrow 0 ; \quad I+E_{k} \rightarrow I$ as $I \rightarrow \infty$. Therefore, for sufficiently large $\mathrm{k},\left(I+E_{k}\right)$ cannot be singular. Hence it has QR decomposition and we may write

$$
I+E_{k}=Q_{E}^{k} R_{E}^{k}
$$

where $Q_{E}^{k}, R_{E}^{k}$ are unitary and upper triangular respectively. It can be shown that $Q_{E}^{k} \rightarrow I$ and $R_{E}^{k} \rightarrow I$ as $E_{k} \rightarrow 0$, and we sketch here the proof*. Multiply (17) by $\left(Q_{E}^{k}\right)^{H}$, we get $\left(Q_{E}^{k}\right)^{H}+G_{k}=R_{E}^{k}$. Where $G_{k}=\left(Q_{E}^{k}\right)^{H} E_{k} \rightarrow 0$ together with $E_{k}$ as $\mathrm{k} \rightarrow \infty$. By an argument, similar to the uniqueness proof of the QR decomposition,

$$
\left(R_{E}^{k}\right)^{H} R_{E}^{k}=I+H_{k}
$$


where $H_{k}=Q_{E}^{k} G_{k}+G_{k}^{H}\left(Q_{E}^{k}\right)^{H}+G_{k}^{H} G_{k} \rightarrow 0$ as $\mathrm{k} \rightarrow \infty$, and thus $R_{E}^{k}$ is "near unitary" for large k. For simplicity's sake, let us consider the $(3,3)$ case, as (nxn) case can be handled similarly. Let $R_{E}^{k}$ and $H_{k}$ be given by:

$$
R_{E}^{k}=\left(\begin{array}{ccc}
r_{11} & r_{12} & r_{13} \\
0 & r_{22} & r_{23} \\
0 & 0 & r_{33}
\end{array}\right) \text {, and } H_{k}=\left(\begin{array}{lll}
h_{11} & h_{12} & h_{13} \\
h_{21} & h_{22} & h_{23} \\
h_{31} & h_{32} & h_{33}
\end{array}\right)
$$

Then (18) implies the following system of equations: $r_{11} r_{11}=1+h_{11}$, where the bar above $\mathrm{r}$ denotes the conjugate.

$$
\begin{aligned}
& r_{11} r_{12}=h_{12} \\
& r_{11} r_{13}=h_{13} \\
& -{ }^{-} \\
& r_{11} r_{12}=h_{21}
\end{aligned}
$$

Note: * Publications used, as source for this manuscript did not discuss this point. 


$$
\begin{aligned}
& r_{12} r_{12}+r_{22} r_{22}=1+h_{22} \\
& -- \\
& r_{12} r_{13}+r_{22} r_{23}=h_{23} \\
& - \\
& r_{13} r_{11}=h_{31} \\
& --r_{13} r_{12}+r_{23} r_{22}=h_{32} \\
& --{ }_{-} r_{13}+r_{23} r_{23} r_{33} r_{33}=1+h_{33}
\end{aligned}
$$

If each $r_{i j} \rightarrow 0$ with $\mathrm{k} \rightarrow \infty$ and if $r_{i i}$ are chosen to be positive, then it can be shown recursively that

$$
\begin{aligned}
& r_{i i} \rightarrow 1 \\
& \text { and } \quad \text { if } \mathrm{i} \neq \mathrm{j} \text { then, } \\
& r_{i j} \rightarrow 0,
\end{aligned}
$$

Thus the matrix $R_{E}^{k} \rightarrow I$ with $\mathrm{k} \rightarrow \infty$. From (17), if $R_{E}^{k} \rightarrow I$ and $E_{k} \rightarrow 0$ as $\mathrm{k} \rightarrow \infty$, then $Q_{E}^{k} \rightarrow I$ as $\mathrm{k} \rightarrow \infty$. We will now return to the proof of the QR method. From (16) and (17), $\xi_{k} \mathcal{R}_{k}=Q_{x}\left(Q_{E}^{k} R_{E}^{k}\right) R_{x} D^{k} R_{y}$.

We proved at the beginning of this section that the decomposition given be (8) is unique if we select the diagonal element of $R_{k}(k=1,2,3, \ldots \ldots \ldots . .$.$) to be positive.$ Therefore 
$\xi_{k}=Q_{x} Q_{E}^{k}$. For sufficiently large $\mathrm{k}, Q_{E}^{k} \rightarrow I$. Therefore $\xi_{k} \rightarrow Q_{x}$, thus $\xi_{k}$ tends to $\rightarrow Q_{x}$, as $\mathrm{k} \rightarrow \infty$. From (10), and (12),

$$
A_{k+1}=\xi_{k}^{H} A_{1} \zeta_{k} \rightarrow Q_{x}^{H} X D X^{-1} Q_{x}=Q_{x}^{H} X\left(P^{T} P\right) D\left(P^{T} P\right) X^{-1} Q_{x}
$$

Using the decomposition of $\left(X P^{T}\right)$ from (14), we get $A_{k+1} \rightarrow Q_{x}^{H} Q_{x} R_{x}\left(P D P^{T}\right) R_{x}^{-1} Q_{x}^{H} Q_{x}=R_{x} \tilde{D}^{R_{x}^{-1}}$. Where $\tilde{D}=P D P^{T}$. Hence the matrix $A_{k}$ converges indeed to an upper triangular matrix.

Algorithm: Each step of the QR algorithm requires the QR factoring of $A_{k}(k=1,2,3 \ldots \ldots . . .$.$) . For theoretical purposes it was convenient to think of this in$ terms of the Schmidt ortho-normalization of the column of $A_{k}$. In practice, due to round off error, this commonly gives rise to a $Q_{k}$ which is by no means orthogonal [12, p. 243]. In such cases the numerical stability associated with unitary similarity transformation could be lost. In practice the matrix $Q_{k}^{H}$ is determined such that

$$
Q_{k}^{H} A_{k}=R_{k} \text {. }
$$

The matrix $Q_{k}^{H}$ is determined as the product of plane rotations by Given's method [6], or by the use of unitary matrices using Householders method. Here only Householder's triangularization will be discussed briefly. 
In the Householder's method, the matrix $Q_{k}^{H}$ is obtained as the product of elementary unitary matrices of the form

$$
u=I-2 w w^{H}
$$

Here $w$ is a column vector such that

$$
w^{H} w=1 .
$$

Further using (19), we have, $u^{H} u=\left(I-2 w w^{H}\right)\left(I-2 w w^{H}\right)=I$,

that is, $u$ is unitary. Let $B$ denote a $(\mathrm{n}, \mathrm{n})$ matrix. Householder's method is a finite iteration consisting of (n-1) steps. Let $B_{r}$ be the $\mathrm{r}^{\text {th }}$ step of Householder's technique to factor $B$ as QR. On the completion of the $\mathrm{r}^{\text {th }}$ step, $B$ is already upper triangular in its first r columns, we may write

$$
B_{r}=\left(\begin{array}{cc}
R_{r} & v_{r} \\
\underset{r}{0} & \underbrace{w_{n-r}}_{n-r}\} n-r
\end{array}\right)
$$

where $R_{r}$ is an upper triangular matrix of order $\mathrm{r}, v_{r}$, and $w_{n-r}$ are of orders as shown in the diagram.

In the $(\mathrm{r}+1)^{\text {th }}$ step, we used a matrix defined by (19) and of the form

$$
u_{r}=\left(\begin{array}{cc}
I & 0 \\
0 & I-2 w w^{H}
\end{array}\right)
$$


Thus the vectors $w$ in $(20)$ are constructed so that the $(\mathrm{r}+1)^{\text {th }}$ step, the first $\mathrm{r}$ components of $w$ are zero. The $(\mathrm{r}+1)^{\text {th }}$ step is given by

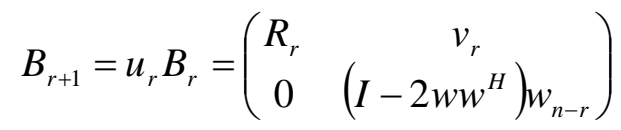

so that only $w_{n-r}$ is modified. $w$ is chosen so that the first column of $\left(I-2 w w^{H}\right) w_{n-r}$ is null except its first element. Therefore, at the end of $(\mathrm{n}-1)^{\text {th }}$ steps,

$$
u_{n-1} \cdots . . . u_{1}=B_{n-1} \text {. }
$$

The product of unitary matrices is unitary and $B_{n-1}$ is upper triangular.

$$
u_{n-1} \ldots . . . u_{1}=Q^{H} \text { and } B_{n-1}=R \text {. }
$$

Therefore, $Q^{H} B=R$ or $B=Q R$. Thus the QR factoring of a matrix $B$ is achieved.

Rate of Convergence: In the QR method, the sub-diagonal elements (i, j) of $A_{k}(k=1,2,3, \ldots .$.$) converge to zero as fast as \left(\frac{\lambda_{i}}{\lambda_{j}}\right)^{k}$. The convergence could be slow if the separation of the eigenvalues is poor.

Application and Limitations: The decomposition of $A$ into QR is too laborious (requiring $0\left(\mathrm{n}^{3}\right)$ operations) for full matrices. For this reason, the matrix is first 
transformed into Hessenberg form. It can be shown that the Hessenberg form is invariant with respect to the QR transformation [12, p.502].

The great benefit the QR with respect to LR transformation is that, because of the use of unitary transformation it is very stable numerically.

A considerable economy in the total number of computation can be obtained by using the following technique. If during the course of the iteration, the magnitude of any sub-diagonal element in position $(\mathrm{i}, \mathrm{i}+1)$ does not exceed a tolerance $\varepsilon$, the eigenvalue problem of $A$ is reduced in approximation to that of matrix $A_{11}, A_{22}$ of order i, n-i, respectively, as shown for a matrix $A_{k}$.

$$
A_{k}=\left(\begin{array}{ccc}
A_{11} & \left.A_{12}\right\} r \\
0 & \varepsilon & \\
\underbrace{0}_{r-1} & \underbrace{0}_{1} & \underbrace{A_{22}}_{n-r}\} n-r
\end{array}\right)
$$

The eigenvalues of $A_{k}$ are approximated by the eigenvalues of $A_{11}$, and $A_{22}$. If the sub-diagonal element (not exceeding $\varepsilon$ ) happens to be in the position (n, n-1), then $\left(A_{k}\right)_{n n}$ may be regarded as an eigenvelue and deflation to a Hessenberg matrix of order (n-1) may be achieved by dropping the last row and column. If it is in the (n-1, $n-2)$ position, then the eigenvalue of the $(2,2)$ matrix in the lower right hand corner 
may be regarded as the eigenvalues of the original matrix, and deflation to a Hessenberg matrix of order (n-2) may be achieved [12, p. 126].

The rate of convergence of the QR method is better by shifting the origin at each iteration, as discussed in connection with the power method. Arithmetic throughout the process can be maintained in real numbers by combining two origin shifts or a pair of complex conjugate origin shifts. An algorithm treating the QR process can be found in [10].

Oepomo's Iteration for Eigenvalues. Let A be an n x n essentially positive matrix. The algorithm can be used to numerically determine the eigenvalue $\lambda_{A}$ with the largest real part and the corresponding positive eigenvector $x[A]$ for essentially positive matrices. This algorithm is based on previous manuscript $[8,9]$. A matrix $\mathrm{A}=\left(a_{i j}\right)$ is an essentially positive matrix if $a_{i j} \geq 0$ for all $i \neq j, 1 \leq i, j \leq n$, and A is irreducible.

Let $x>0$ be any positive $n$ components vector [8]. Let

$$
\begin{aligned}
& Z_{i}(x)=\sum_{\substack{r=1 \\
r \neq i}} a_{i r} x_{r} ; \\
& f_{i}(x)=\frac{(A x)_{i}}{x_{i}} \equiv \sum_{j=1}^{n} \frac{a_{i, j} x_{j}}{X_{i}}(i \in N) ;
\end{aligned}
$$


$m(x)=\min _{i \in N} f_{i}(x)$;

$M(x)=\max _{i \in N} f_{i}(x) ;[2,8$, and 9$]$

$\Delta(x)=M(x)-m(x)$

$\|x\|=\sum_{i=1}^{n} x_{i}$

The following theorem is an application of corollary 2.3 from [8] to the design of algorithms using the Perron-Frobenius-Collatz minimax principle for the calculation $x[A]$. Let $\left\{x^{p}\right\}(\mathrm{p}=0,1,2, \ldots .$.$) be a sequence of positive vectors and$ $x^{p}=\left[x_{1}^{p}, x_{2}^{p}, \ldots \ldots \ldots, x_{n}^{p}\right]^{T}$.

Theorem 4 If the sequence $\left\{x^{p}\right\}(p=0,1,2, \ldots$.$) of positive unit vectors is such that$ either $\quad m\left(x^{p}\right) \rightarrow \Lambda[A] \equiv \lambda_{A} \quad$ or $\quad M\left(x^{p}\right) \rightarrow \Lambda[A] \equiv \lambda_{A} \quad$ as $\quad p \rightarrow \infty \quad$ then $x^{p} \rightarrow x[A] \equiv \xi$. Moreover, the sequence $\left\{m\left(x^{i}\right)\right\},\left\{x^{i}\right\}$ are equi-convergent in the sense that an index $v$ and a constant $K>0$ exist such that $\left\|x^{i}-\xi\right\|<K\left[\lambda-m\left(x^{i}\right)\right]$ if $i \geq v$. Similar statements can be expressed if $M\left(x^{i}\right) \rightarrow \Lambda[A] \equiv \lambda_{A}$ is known. Proof: [8, theorem 2.4, and 9, theorem 1].

\section{Numerical Implication of Theorem 4.}

We will now define a group of sequences, the "decreasing-sequence" which will defined later. 
Decreasing-sequence: Let $Y^{r}(x)(\mathrm{r}=1,2,3, \ldots . ., \mathrm{n})$ be an $\mathrm{n}$ component vector valued function such that the following equation is valid:

$Y_{i}^{r}(x)=x_{i} \quad$ if $\mathrm{i} \neq \mathrm{r}$, or $\Omega_{r}(x)$ if $\mathrm{i}=\mathrm{r}$.

Here $\Omega_{r}(x)$ (r=1, 2, 3, ...., n) are scalar valued functions which are having properties as follows:

a) $\Omega_{r}(x)$ is a continuous positive valued functions which maps the set of positive vectors $V_{+}$into a set of real numbers $\mathrm{R}$.

b) $\Omega_{r}(x) \leq x_{r}$.

c) $f_{r}\left(Y^{r}(x)\right) \leq M(x)$.

d) Equality in c) may be applicable only if $Y^{r}\left(x^{r-1}\right)$ converges to $x$; this yields that $f_{r}(x)=M(x)$.

e) If for some $\mathrm{x}>0 \Omega_{r}(x)=x_{r}$ for each $\mathrm{r} \in(\mathrm{N}=1,2,3, \ldots ., \mathrm{n})$ then $f_{1}(x)=f_{2}(x)=\ldots \ldots \ldots \ldots=f_{n-1}(x)=\lambda$. This will imply that $f_{n}(x)=\lambda$ according to Collatz, hence $\mathrm{x}=x[A]$.

Then n-component vector valued function $Y^{r}(x)$ defined in (6) will be referred to as the Decreasing-functions. A sequence $\left\{x^{p}\right\}(\mathrm{p}=0,1,2,3, \ldots .$.$) of positive n-vectors$ 
is constructed which satisfy the conditions of theorem 1 . The terms of the sequence $\left\{x^{p}\right\}$ are generated by the following recursive formula:

$$
x^{p+1}=Y^{p+1}\left(x^{p}\right)
$$

Where $Y^{k}(x)=Y^{k+n}(x) \quad(\mathrm{k}=1,2,3, \ldots)$. If $x^{0}$ is given the sequence $\left\{x^{p}\right\}$ is completely defined. $x^{p+1}$ and $x^{p}$ differ only in the $\mathrm{r}^{\text {th }}$ component where

$$
r \equiv p+1 \text { (mode } \mathrm{n})
$$

Such a sequence will be called a decreasing maximum ratio sequence or briefly decreasing-sequence.

Corollary 1: Any decreasing-sequence converges to $x_{A}$. Proof: see [9], and Theorem 4 on this manuscript.

We will now define a second group of sequences, the "Increasing-sequence". Increasing-sequence: Let $y^{r}(x)(\mathrm{r}=1,2,3, \ldots ., \mathrm{n})$ be an $\mathrm{n}$ component vector valued function such that the following equation is valid:

$$
\begin{array}{r}
y_{i}^{r}(x)=x_{i} \quad \text { if } \mathrm{i} \neq \mathrm{r} \\
\omega_{r}(x) \text { if } \mathrm{i}=\mathrm{r} .
\end{array}
$$

Here $\omega_{r}(x)(\mathrm{r}=1,2,3, \ldots . ., \mathrm{n})$ are scalar valued functions which are having properties as follows: 
f) $\omega_{r}(x)$ is a continuous positive valued function and bounded in $\sum_{r}=\left(\mathrm{x} \mid z_{r}(x)\right.$ $>0 ; \mathrm{x} \geq 0)$.

g) $\omega_{r}(x) \geq x_{r}$

h) $\quad f_{r}\left(y^{r}(x)\right) \geq m(x)$

i) Equality in h) may be applicable only if $y^{r}\left(x^{r-1}\right)$ converges to $x$; this yields that $f_{r}(x)=m(x)$.

j) If for some $\mathrm{x}>0 \omega_{r}(x)=x_{r}$ for each $\mathrm{r} \in(\mathrm{N}=1,2,3, \ldots \ldots, \mathrm{n})$ then

$f_{1}(x)=f_{2}(x)=\ldots \ldots \ldots \ldots . . .=f_{n-1}(x)=\lambda$. This will imply that $f_{n}(x)=\lambda$ according to Collatz, hence $\mathrm{x}=x[A]$.

Then n-component vector valued function $Y^{r}(x)$ defined in (9) will be referred to as the Decreasing-functions. A sequence $\left\{x^{p}\right\}(p=0,1,2,3, \ldots . .$,$) of positive n-vectors$ is constructed which satisfy the conditions of theorem 1 . The terms of the sequence $\left\{X^{p}\right\}$ are generated by the following recursive formula:

$$
x^{p+1}=y^{p+1}\left(x^{p}\right)
$$


where $y^{k}(x)=y^{k+n}(x)$ and $y(x)$ is as defined in (9) $(\mathrm{k}=1,2,3, \ldots)$. If $x^{0}$ is given the sequence $\left\{x^{p}\right\}$ is completely defined. Such a sequence will be called an increasing minimum ratio sequence or briefly increasing-sequence.

Corollary 2: Any Increasing-sequence converges to $x_{A}$.

Proof: see proof in [9], and Theorem 4 on this manuscript.

Numerical tests indicate that a simultaneous application of the decreasing and increasing sequences will converge faster than either the decreasing or increasing sequence separately. Therefore, we will define a sequence of vectors $\left\{x^{p}\right\}$ which are constructed by alternating methods of the decreasing or increasing type functions.

We will describe a sequence of $n$ steps which generate $x^{j+1} \ldots \ldots . . x^{j+n}(j=0, n, 2 n$, $\ldots . .$.$) in an iteration. If the decreasing functions \left(y^{r}(x), r=1,2, \ldots \ldots, n\right)$ are used to generate the $n$ terms of the sequence $\left\{x^{p}\right\}$ during iteration as defined in (8) then we will say that the iteration is in decreasing mode. Similarly the iteration is in the increasing mode if increasing functions are used as defined in (12). Successive terms of the sequence $\left\{x^{p}\right\}$ can be defined recursively in the following respects:

$$
x^{k+1}=Y^{k+1}\left(x^{k}\right) \quad \text { for } \mathrm{k}=0,1,2, \ldots \ldots
$$


or

$$
x^{k+1}=y^{k+1}\left(x^{k}\right) \quad \text { for } \mathrm{k}=0,1,2, \ldots \ldots
$$

Where $k=0$ corresponds to the input vector. The first iteration could be either in the increasing or in the decreasing mode.

We also define the sequence of real number $\left\{t_{\ell}\right\} \quad\left\{T_{\ell}\right\}$ as follows:

$$
t_{0}=m\left(x^{0}\right) . \quad T_{0}=M\left(x^{0}\right) .
$$

At the end of each iteration we consider the following inequalities:

$$
m\left(x^{n \bullet \ell}\right) \geq t_{\ell-1}
$$

and

$$
M\left(x^{n \bullet \ell}\right) \leq T_{\ell-1}
$$

Where $(\ell=1,2,3 \ldots . .$.$) are indexes of the iteration. If inequalities (34-1) and (34-2)$ are met, we may set

$$
\begin{aligned}
& t_{\ell}=m\left(x^{n \bullet \ell}\right) \\
& T_{\ell}=M\left(x^{n \bullet \ell}\right)
\end{aligned}
$$

and the mode of the next $(\ell+1)^{\text {st }}$ iteration will be different from the $\ell^{\text {st }}$ iteration, i.e. if the $\ell^{s t}$ iteration is an increasing sequence then the $(\ell+1)^{\text {st }}$ iteration is a decreasing sequence or vice versa. If either inequality (34-1) or (34-2) is not satisfied then the 
mode or direction of the $(\ell+1)^{\text {st }}$ iteration is the same as that of the $\ell^{\text {st }}$ iteration and we set:

$$
t_{\ell}=t_{\ell-1} \quad \text { And } \quad T_{\ell}=T_{\ell-1} \text {. }
$$

A sequence having the above-mentioned properties will be called Oepomo's alternating sequence iteration.

Corollary 3: Any Oepomo's alternating sequence iteration converges to $x_{A}$.

Proof: see Corollaries 1 and 2, and Theorem 4 on this manuscript.

Corollary 1, 2, and 3 described above lay the foundation of the procedure of an iterative algorithm for the determination of the positive eigenvector of essentially positive matrices. The choice of the functions $\Omega_{r}(x), \omega_{r}(x)$ is open but is subject to the restrictions specified in connection with the decreasing and increasing sequences. In theorem 5 and theorem 6 which follow, a possible choice for $\Omega_{r}(x)$ and $\omega_{r}(x)$ is given.

Theorem 5 Let $H_{r}(x)(r=1,2,3, \ldots . ., n)$ denote continuous, positive valued functions which map the set of positive vectors $V_{+}$into a set of real number $R$ such that

$$
m(x) \leq H_{r}(x) \leq M(x)
$$


and equality may hold on either side of (16) only if $m(x)=M(x)=\lambda_{A}$.

For $r \varepsilon N(N=1,2,3, \ldots . ., n)$, let

$$
\begin{aligned}
\Omega_{r}(x) & =x_{r} & & \text { if } f_{r} \geq H_{r} \\
& =\frac{z_{r}}{H_{r}-a_{r r}} & & \text { if } f_{r}<H_{r}
\end{aligned}
$$

Where $H_{r} \equiv H_{r}(x), f_{r} \equiv f_{r}(x)$, and all notations are defined in $(1,2,3,4$, and 5). Then the functions $\Omega_{r}(x)$ (together with a starting vector $x^{0}$ ) define $a$ decreasing sequence.

Proof: see [9]

Theorem 6 Let $h_{r}(r=1,2,3, \ldots . . ., n)$ denotes a continuous bounded function mapping $\sum_{r} \rightarrow R$, such that $m(x) \leq h_{r}(x) \leq M(x)$

and equality may hold on either side of (24) only if $m(x)=M(x)=\lambda_{A}, x=x_{A}$, ( $\sum_{r}$ has been introduced in equation (21). We further define

$$
\begin{array}{rlrl}
W_{r}(x)=x_{r} & & \text { if } f_{r} \leq h_{r} \\
& =\frac{z_{r}}{h_{r}-a_{r r}} & & \text { if } f_{r}>h_{r}>\frac{z_{r}}{\|x\|}+a_{r r} \\
& =\|x\| & & \text { otherwise. }
\end{array}
$$


Then the function $w_{r}$ (together with an $x^{0}>0$ ) define an increasing sequence.

Proof: see [9]

The requirements of functions $H_{r}(x)$, and $h_{r}(x)$

The functions $H_{r}(x)$, and $h_{r}(x)$ can be selected in many means. The following are a few of the possible choices:

a) $h_{r}(x)=\frac{1}{2}[M(x)+m(x)]=\mu(x) ; H_{r}(x)=\frac{1}{2}\left[M^{*}(x)+m(x)\right]=\mu^{*}(x)$ where $r \in \mathrm{N}$ $(\mathrm{N}=1,2,3, \ldots ., \mathrm{n})$ and $M^{*}(x)=\min \left(m(x), M_{1}\right)$ and $M_{1}$ is an upper estimate of the eigenvalue $\lambda_{A}$, e.g., $M_{1}=M\left(x^{0}\right)$. In [3], $\mathrm{m}(\mathrm{x})$ is defined as $\operatorname{Min}_{i \in k} R_{k}(x)$ and $\mathrm{M}(\mathrm{x})$ is defined as $\operatorname{Max}_{i \in k} R_{k}(\mathrm{x})$.

b) For full matrices a reasonable choice for $H_{r}(x)$, and $h_{r}(x)$ are derived from the arithmetic mean of the $f_{i}{ }^{\prime} s . H_{r}(x)=\sigma(x)=\frac{1}{n} \sum_{i=1}^{n} f_{i}(x)$; and $h_{r}(x)=\sigma^{*}(x)=\frac{1}{n} \sum_{i=1}^{n} f_{i}^{*}(x)$ where $f_{i}^{*}(x)=\min \left(f_{i}(x), M_{1}\right)$.

c) Another simple choice for $h_{r}(x) ; H_{r}(x)$ is a weighted arithmetic mean of $f_{i}$ 's : 
$h_{r}(x)=H_{r}(x)=\frac{\sum_{i=1}^{n} f_{i}(x) x_{i}}{\|x\|}=v(x) . \quad v(x)$ can also be defined in the following mean:

$$
v(x)=\frac{\sum_{i=1}^{n} b_{i} x_{i}}{\|x\|}
$$

where $\quad b_{i}=\sum_{j=1}^{n} a_{j i}$

Algorithm: A step of the alternating sequence iteration algorithm consists in modifying a single component $x_{r}$ of $\mathrm{x}$. As a result $z_{i}, f_{i},\|x\|, v(x)$ will have to be calculated at each iteration. Calculating $z_{i},\|x\|$ from their definition in equations (21), and (25) will be referred as recalculating. A considerable reduction of calculation can be accomplished if instead of recalculating these terms are merely updated according to the following steps:

$$
\begin{array}{ll}
\left\|x^{p+1}\right\|=\left\|x^{p}\right\|+\left(x_{r}^{p+1}-x_{r}^{p}\right) & \\
z_{i}^{p+1}=z_{i}^{p}+a_{i r}\left(x_{r}^{p+1}-x_{r}^{p}\right) & \mathrm{I}=1,2,3, \ldots ., \mathrm{n} \\
v\left(x^{p+1}\right)=v\left(x^{p}\right)+b_{r}\left(x_{r}^{p+1}-x_{r}^{p}\right) . &
\end{array}
$$

These steps will be referred to as the updating iteration. The updating equations can be obtained easily from equations 21 through 25 . To prevent the 
accumulation of round off errors, after a number of iterations the variables will have to be recalculated instead of updating. If we are working in a double precision, our previous experiences indicate that it is more than sufficient to recalculate after every twenty five iterations.

Over-Relaxation Method: From various choices for functions $H_{r}(x), h_{r}(x)$, and $v(x)$ as indicated in equation (34) seems to give a rapid convergence at least for full matrices. The purpose of this section is to present a variant of equation (40) by introducing the over-relaxation technique. We consider the following equation

$$
h_{r}(x)=(1-\gamma) f_{r}(x)+(\gamma) v(x)
$$

As it well known, for several suitable values for $\gamma$, is the over-relaxation factor, and $1 \leq \gamma<2$. Equation (36) may be useful in case of banded matrices. The over-relaxation method contains the following cases:
a) $\quad \gamma=1$ for simultaneous over-relaxation method, and
b) $1<\gamma<2$ for over-relaxation method.

Error Vector In all methods the quantity $\Delta(x)=M(x)-m(x)$ as indicated in (4) is used as a measure of accuracy. 
Discussions Before we go any further the following issues should be understood. Are both eigenvalues and eigenvectors required to be calculated, or are eigenvalues by itself enough? Is only the absolute largest eigenvalue of interest? Does the matrix have special characteristics, such as real symmetric, essentially positive and so on? If all eigenvalues and eigenvectors are required then this algorithm can not be used;

If a matrix (A) is essentially positive and the positive eigenvector $\left(x_{A}\right)$ and the corresponding eigenvalue $\left(\lambda_{A}\right)$ are of particular interest, then the algorithm can be used. Each step of the algorithm requires $n^{2}+0(n)$ computations, if the parameters are chosen for the best rate of convergence. It is possible to assume that in half the steps practically no computations are needed, resulting thereby in $\frac{n^{2}}{2}+0(n)$ computations for each iteration. As previously stated, after some iteration the variables will have to be recalculated instead of updating. Recalculations need $n^{2}+0(n)$ additional computations. If the computations are performed in double precision, recalculation will not have to be performed so often. As a result recalculations do not increase the total number of computation significantly. 
For our numerical comparisons all three algorithms, Power, Oepomo, and QR methods, were tried to solve eigenvalue of the following matrices:

a) All three algorithms were used to estimate the eigenvalue of Hilbert matrices of various orders. Let $H_{n}$ be a Hilbert matrix of order n. The elements of Hilbert matrix are defined according to the following relation:

$$
a_{i j}=\frac{1}{(i+j-1)} \quad 1 \leq \mathrm{i}, \mathrm{j} \leq \mathrm{n}
$$

The results of the 3 methods can be seen in Tables I, II, and III.

b) We would like to find the efficiency of the three algorithms, when a matrix had eigenvalues of nearly the same modulus. So it was decided to pick a matrix of order $\mathrm{n}$ that was almost cyclic $\left(c_{n}\right)$. Consider the below mentioned matrix

$$
\left[\begin{array}{ll}
A_{1,1} & A_{1,2} \\
A_{2,1} & A_{2,2}
\end{array}\right]
$$

The elements of $A_{1,2}$ and $A_{2,1}$ were defined as follows

$$
a_{i, j}=\frac{1}{(i+j-1)}, A_{1,2} \text { is a }(8,12) \text { matrix, and } A_{2,1} \text { is a }(12,8) \text { matrix. }
$$

The elements of $A_{1,1}$ and $A_{2,2}$ were defined in the following respects 


$$
a_{i, j}=\frac{10^{-2}}{(i+j-1)}, A_{1,1} \text { is a }(12,12) \text { matrix, and } A_{2,2} \text { is a }(8,8) \text { matrix. }
$$

If the elements of $A_{1,1}$ and $A_{2,2}$ were replaced by zero then the matrix would be nearly cyclic.

For comparisons, the results of those 3 methods can be seen in Tables IV, V, and VI.

c) Introducing a proper shift of origin could speed up the convergence of power algorithm [9]. So it was decided to try to that kind of matrix such that by introducing a shift of origin would not help the speed of convergence. Such a matrix of order $n\left(Q_{n}\right)$ can be given by the following relations

$a_{i, j}=\max \left[\frac{n-i}{n}, \frac{n-j}{n}\right]$ for $1 \leq \mathrm{i}, \mathrm{j} \leq \mathrm{n}, \& a_{i, i}=\left(\frac{n-i}{n}\right)-\left(\frac{50\left(i-\frac{n+1}{2}\right)}{n}\right)$ for $1 \leq \mathrm{i} \leq \mathrm{n}$

The results of our tests are indicated in Tables VII, VIII, and IX.

We will here assume that we are interested in the positive eigenvector and the corresponding eigenvalue of the essentially positive matrix. From our trials, it is obvious that in all three cases the rate of convergence of our new algorithm is better or at least as fast as the power [4]. The QR [13] algorithm converges very slowly in the last two cases, when the separation between the eigenvalues is poor. Let us 
consider the results of case b, when the matrix is nearly cyclic. For a cyclic matrix there are some eigenvalues of equal modulus, and so for a matrix that is "near cyclic” it is plausible to assume the separation between the modules is very poor. The new algorithm takes about 5700 multiplication and divisions to reach an accuracy of 8 digits; which is about 5 times the computations of the power algorithm and the QR algorithm reach an accuracy of 2 digits and 4 digits respectively. We should remember that the QR algorithm is not specifically designed to calculate just one eigenvalue; therefore, a comparable efficiency cannot be expected. Thus from our recent experience we can conclude that the new method shows a good speed of convergence even when the separation of the eigenvalues is poor. However in the case of banded matrices the new algorithm converges slowly. The new algorithm was tried on various banded matrices arising from finite difference approximation to boundary value problems of ordinary differential equations. A computer code was written specially for banded matrices, to take advantage of the large number of zero elements in a banded matrix. We will here summarize the results of our computer runs with the following $(20,20)$ matrix

$$
\begin{array}{ll}
a_{i i}=-2 & \text { if } 1 \leq \mathrm{i} \leq \mathrm{n} . \\
a_{i+1, i}=a_{i, i+1}=1 & 1 \leq \mathrm{i} \leq \mathrm{n}-1 .
\end{array}
$$




$$
a_{i, j}=0 \quad \text { otherwise }
$$

The over relaxation method as described in (36), was tried on the above-mentioned matrix with values of $\gamma$ ranging from 1 to 1.99. The speed convergence did not show a remarkable dependence of $\gamma$. An 8 digit of accuracy was obtained in 168 iterations for $\gamma=1.73$ whereas for full matrices the new algorithm gave a 9 digit of accuracy in 21 steps.

We will now return our attention to full matrices. Let $R_{n}$ be a matrix (of order $\mathrm{n}$ ) with pseudo-random entries. The new algorithm and the power algorithm were tried on each family of matrices $\left(R_{n}, C_{n}, H_{n}\right)$ of order $\mathrm{n}=20,40$, and 80 . The speed of convergence is almost the same for the two algorithms remembering that each iteration step of the power algorithm requires about twice as many computations. Within one algorithm it is somewhat surprising that the number of iteration steps required for as given accuracy hardly depend on the order of the Hilbert matrix at all. Acknowledgment The author wishes to thank Prof. Schneider, Mathematics Department at the University of Wisconsin in Madison, for his suggestions during the writing of the author's earlier manuscripts $[8,9]$ and criticism during the writing. His criticism and suggestions yield the development of this survey. The author acknowledges indebtedness to referees, and to their stimulating comments during the review of the earlier articles $[8,9]$. 
TABLE I

Hilbert Matrix $H_{40}$ Power Method Method

\begin{tabular}{|l|l|l|}
\hline Operations & $\Delta(x)$ & $\log \Delta(x)$ \\
\hline 1640 & 1.423 & 0.3528 \\
\hline 4921 & $1.41 \times 10^{-1}$ & -1.958 \\
\hline 8200 & $1.39 \times 10^{-2}$ & -4.275 \\
\hline 11490 & $1.3441 \times 10^{-3}$ & -6.611 \\
\hline 14764 & $1.296 \times 10^{-4}$ & -8.949 \\
\hline 18040 & $1.25 \times 10^{-5}$ & -11.288 \\
\hline 21322 & $1.208 \times 10^{-6}$ & -13.626 \\
\hline 24600 & $1.116 \times 10^{-7}$ & -15.965 \\
\hline 27880 & $1.123 \times 10^{-8}$ & -18.304 \\
\hline
\end{tabular}

TABLE III

Hilbert Matrix $H_{40}$ QR Method

Method

\begin{tabular}{|l|l|l|}
\hline Operations & $\Delta(x)$ & $\log \Delta(x)$ \\
\hline 17400 & $2.423 \times 10^{-2}$ & -3.21 \\
\hline 23400 & $1.007 \times 10^{-4}$ & -9.202 \\
\hline 26600 & $8.263 \times 10^{-9}$ & -18.614 \\
\hline 332000 & $2.422 \times 10^{-2}$ & -3.722 \\
\hline 442000 & $1.0096 \times 10^{-4}$ & -9.212 \\
\hline 506000 & $8.239 \times 10^{-9}$ & -18.615 \\
\hline
\end{tabular}

TABLE II

Hilbert Matrix $H_{40}$ Oepomo

\begin{tabular}{|l|l|l|}
\hline Operations & $\Delta(x)$ & $\log \Delta(x)$ \\
\hline 4800 & $1.016 \times 10^{-1}$ & -2.288 \\
\hline 9600 & $3.71 \times 10^{-3}$ & -5.597 \\
\hline 14400 & $8.549 \times 10^{-5}$ & -9.368 \\
\hline 19200 & $3.068 \times 10^{-6}$ & -12.693 \\
\hline 24000 & $7.061 \times 10^{8}$ & -16.467 \\
\hline 28800 & $2.536 \times 10^{-9}$ & -19.289 \\
\hline 29700 & $9.977 \times 10^{-10}$ & -20.628 \\
\hline
\end{tabular}

TABLE IV

Almost Cyclic Matrix $C_{40}$ Power

\begin{tabular}{|l|l|l|}
\hline Operations & $\Delta(x)$ & $\log \Delta(x)$ \\
\hline 3780 & $3.091 \times 10^{-1}$ & -1.174 \\
\hline 7980 & $2.089 \times 10^{-1}$ & -1.566 \\
\hline 1188 & $1.421 \times 10^{-1}$ & -1.950 \\
\hline 16390 & $9.706 \times 10^{-2}$ & -2.334 \\
\hline 20580 & $6.6381 \times 10^{-2}$ & -2.714 \\
\hline 24780 & $4.5461 \times 10^{-2}$ & -3.092 \\
\hline 28990 & $3.111 \times 10^{-2}$ & -3.478 \\
\hline 33190 & $2.1341 \times 10^{-2}$ & -3.848 \\
\hline 37380 & $1.4651 \times 10^{-2}$ & -4.228 \\
\hline
\end{tabular}


TABLE V

Almost Cyclic Matrix $C_{40}$ Oepomo Method

\begin{tabular}{|l|l|l|}
\hline Operations & $\Delta(x)$ & $\log \Delta(x)$ \\
\hline 1400 & $2.76 \times 10^{-2}$ & -3.589 \\
\hline 2800 & $7.607 \times 10^{-4}$ & -7.187 \\
\hline 4200 & $2.293 \times 10^{-5}$ & -10.699 \\
\hline 5600 & $6.3492 \times 10^{-7}$ & -14.277 \\
\hline 7000 & $1.9246 \times 10^{-8}$ & -17.774 \\
\hline 8400 & $5.3279 \times 10^{-10}$ & -21.358 \\
\hline
\end{tabular}

TABLE VI

Almost Cyclic Matrix $C_{40} \mathrm{QR}$

\begin{tabular}{|l|l|l|}
\hline Operations & $\Delta(x)$ & $\log \Delta(x)$ \\
\hline 2400 & $3.091 \times 10^{-1}$ & -6.622 \\
\hline 3260 & $2.089 \times 10^{-1}$ & -8.308 \\
\hline 4000 & $1.421 \times 10^{-1}$ & -14.178 \\
\hline 44680 & $9.706 \times 10^{-2}$ & -21.798 \\
\hline 21340 & $6.6381 \times 10^{-2}$ & -6.620 \\
\hline 29340 & $4.5461 \times 10^{-2}$ & -8.30892 \\
\hline 37300 & $3.111 \times 10^{-2}$ & -14.178 \\
\hline 41340 & $2.1341 \times 10^{-2}$ & -21.798 \\
\hline
\end{tabular}

TABLE VII

Matrix $Q_{40}$ Power Method

TABLE VIII

Matrix $Q_{40}$ Oepomo Method

\begin{tabular}{|l|l|l|}
\hline Operations & $\Delta(x)$ & $\log \Delta(x)$ \\
\hline 4200 & $6.506 \times 10^{3}$ & 8.788 \\
\hline 3780 & $1.0023 \times 10^{2}$ & 4.608 \\
\hline 7990 & 6.196 & 1.824 \\
\hline 12190 & 1.065 & 0.0609 \\
\hline 16390 & $2.1967 \times 10^{-1}$ & -1.518 \\
\hline 20580 & $4.985 \times 10^{-2}$ & -2.988 \\
\hline 24790 & $1.160 \times 10^{-2}$ & -4.458 \\
\hline 28990 & $2.703 \times 10^{-3}$ & -5.916 \\
\hline 33190 & $6.294 \times 10^{-4}$ & -7.372 \\
\hline
\end{tabular}

\begin{tabular}{|l|l|l|}
\hline Operations & $\Delta(x)$ & $\log \Delta(x)$ \\
\hline 2400 & 3.105 & 1.134 \\
\hline 3260 & $2.089 \times 10^{-4}$ & -8.472 \\
\hline 4000 & $4.778 \times 10^{-7}$ & -14.558 \\
\hline 44680 & $3.124 \times 10^{-10}$ & -21.879 \\
\hline
\end{tabular}


TABLE IX

Matrix $Q_{40}$ QR Method

\begin{tabular}{|l|l|l|}
\hline Operations & $\Delta(x)$ & $\log \Delta(x)$ \\
\hline 4260 & $1.429 \times 10^{1}$ & 2.6591 \\
\hline 5260 & $1.322 \times 10^{-2}$ & -4.329 \\
\hline 5870 & $4.664 \times 10^{-5}$ & -9.9722 \\
\hline 41300 & $1.4294 \times 10$ & 2.6588 \\
\hline 51300 & $1.324 \times 10^{-2}$ & -4.3262 \\
\hline 55300 & $4.668 \times 10^{-5}$ & -9.9722 \\
\hline
\end{tabular}

\section{References}

[1] B. Carnahan, and H. Luther and J. Wilkes, Applied Numerical Methods, John Wiley, Inc., New York, 1969.

[2] L. Collatz. Einschliessungssatz fuer die charakteristischen Zahlen von Matrizen. Math. Z., 48 (1942-43), 221-226.

[3] L. Elsner. Verfahren zur Berechnung des Spektralradius nichtnegativer irreducibler Matrizen II. Computing, 9 (1972), 69-73.

[4] D. K. Faddeev and V. N. Faddeeva. Computational Methods of Linear Algebra, W. H. Freeman and Company, San Fransisco and London, 1973.

[5] J. Francis. The QR Transformation. Comp. Jour., 4 (1961), 265-271. 
[6] C. Froberg. Introduction to Numerical Analysis, Addison-Wesley, Inc., Reading, Massachusetts, 1965.

[7] W. Givens. Computation of Plane Unitary Rotations Transforming a General Matrix to Triangular Form. J. Soc. Industr. Appl. Math Math. , 9 (1958), 26-50.

[8] Tedja S. Oepomo. A Contribution to Collatz's Eigenvalue Inclusion Theorem for Nonnegative Irreducible Matrices. ELA., 10 (2003), 31-45.

[9] Tedja S. Oepomo. Oepomo’s Algorithm for Computing Largest Real Part Eigenvalues of Essentially Positive Matrices: Collatz \& Perron-Frobernius Approach. To be submitted to ELA.

[10] Axel Ruhe. Eigenvalue of a Complex Matrix by the QR Method. BIT. Vol: 6, Number: 4 (1966), 350-358.

[11] R. S. Varga. Matrix Iterative Analysis. Prentice-Hall, Englewood Cliffs, NJ, 1962.

[12] J. H. Wilkinson. The Algebraic Eigenvalue Problem. Claredon Press, Oxford, 1965.

[13] J. H. Wilkinson. Convergence of LR, QR and Related Algorithms. Comp. Jour., 8 (1966), 77.

Received: August 8, 2006 